\title{
AGILE CLIMBING ROBOT DESIGN FOR NDT INSPECTION
}

\author{
Z. ZHAO and G. SHIRKOOHI \\ School of Engineering, \\ London South Bank University, \\ 103 Borough Road, London SE1 0AA \\ United Kingdom
}

\begin{abstract}
Wall climbing robots are extremely useful for deployment in hazardous environments and operation at great heights and other hard to reach space. However, these robots usually require large and heavy suction pads and associated cylinders for supporting the payload and effectual movement during climbing and inspection process and are very difficult to miniaturise. A new type of robot mechanism has been developed where movements are powered by electric motors and hence can be made on a much smaller scale and with the promise of high speed of operation.
\end{abstract}

Keywords: Agile robot, suction pads, electric motors, miniaturisation.

\section{Introduction}

Over the years, a great number of mobile robots have been successfully developed at the Centre for Automated and Robotic Non-Destructive Testing (NDT), London South Bank University (LSBU), UK. Figure 1 shows three inspection robots developed for climbing flat walls and ceiling e.g. large petrochemical storage tanks and ship hulls.

Later, the centre carried out many projects funded by the European Commission. For example, one of the authors has worked on the development of a mobile robot NT systems which can inspect many nozzle joint geometries in radioactive environment as shown in Figure 2, on $860 \mathrm{~mm}$ diameter primary circuit coolant pipe. Figure 2(a) shows this robot for weld inspection in lab tests. Figure 3 shows another mobile robot RobAir developed in the centre for the aircraft fuselage inspection. ${ }^{1}$ All of these robots have the common properties that the movement is actuated by pneumatic cylinders. The advantages of using pneumatic cylinders are high power to weight ratio and quick movement output. Among the disadvantages are difficulties in the design parameters for miniaturisation and achieving reduction in the size of robots, because of the bulk size of cylinders, and difficulties to make precision control due to the nature of the operation of the pneumatic cylinders. This paper will discuss the problems 
associated with the previous version of the mobile robot design and presents the criteria for the design of a new type of climbing robot which overcome the problems and shortcomings of the previous one.

\section{Analysis of the problems associated with the previous robot.}

Using linear cylinders as actuators means that the robot should follow a complex sequence of actions to make a move. Take the Fig 2(b) robot as an example. Here the $\mathrm{y}$-axis represents the front and $\mathrm{x}$-axis represents the right. If the robot wants to move forward, the robot need to switch on the vacuum valves alternatively between the front and back suction cups and the left and right

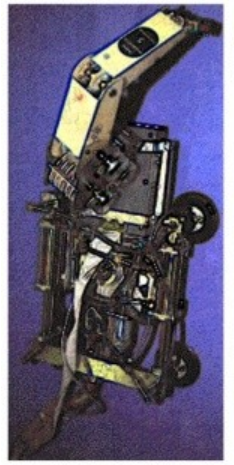

Mass $15 \mathrm{~kg}$

D $490 \times 380 \mathrm{~mm}$ Arm $2 \mathrm{~kg}$

Payload $=15 \mathrm{~kg}$

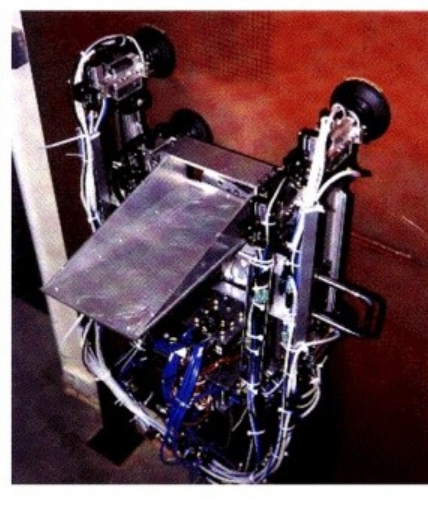

Mass $29 \mathrm{~kg}$

$740 \times 540 \mathrm{~mm}$

Payload $=35 \mathrm{~kg}$

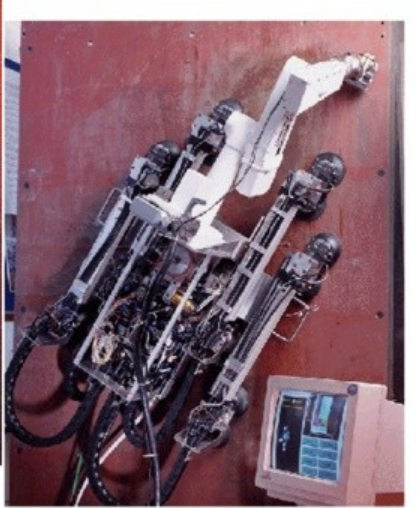

Mass $45 \mathrm{~kg}, 750 \times 700 \mathrm{~mm}, \mathrm{Arm}=13.5 \mathrm{~kg}$

Figure 1 Three climbing robots develop by LSBU in the early years

suction cups to keep the robot adhere to the working surface. At same time, it also needs the leg to lift up or put down to coordinate the move. Figure 4 should then extract codes of the control software which show how to move the robot forward. It can be concluded that such kind a robot needs to go through a complex sequence of movements' control and time delay in between to make it move. It will make the fast and stable movement impossible. RobAir has similar design and has the same problems. 


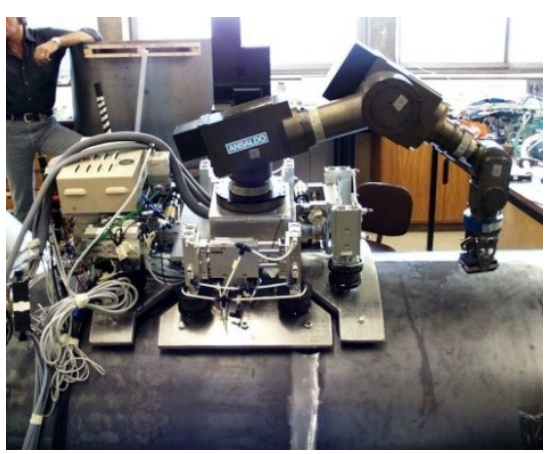

(a) The robot in lab testing

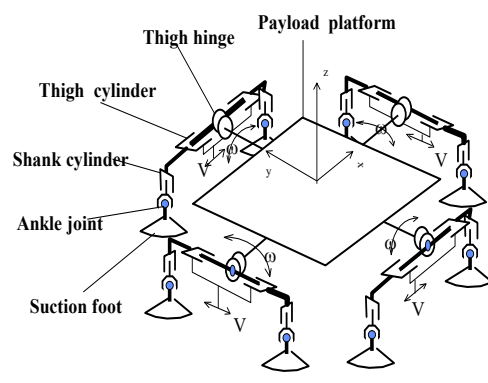

(b) Concept model of robot vehicle

Figure 2 Nuclear Power Plant Robot able to inspect many nozzle joint geometry's in radioactive environment shown on $860 \mathrm{~mm}$ diameter primary circuit coolant pipe.

Large size also means heavy weight. To make the robot able to climbing using vacuum force, special suction cups have been designed for these projects to increase the area of the suction pad and to make it able to accommodate curved surfaces.

For example, RobAir robot uses 36 specially made cups. To arrange these cups to adapt to different kinds of working surface is a sizeable challenge. A stable multi support point structure has been developed to deal with this problem in the RobAir project. ${ }^{1}$ Even with special design, large size is still very difficult to accommodate for different types of surface. For example, if the robot with the length of $2 \mathrm{a}$ works in a curved surface with the radius of curvature of $r$, the difference in the legs will be at least $h=r-\sqrt{ }\left(r^{2}-a^{2}\right)$ compared with the robot
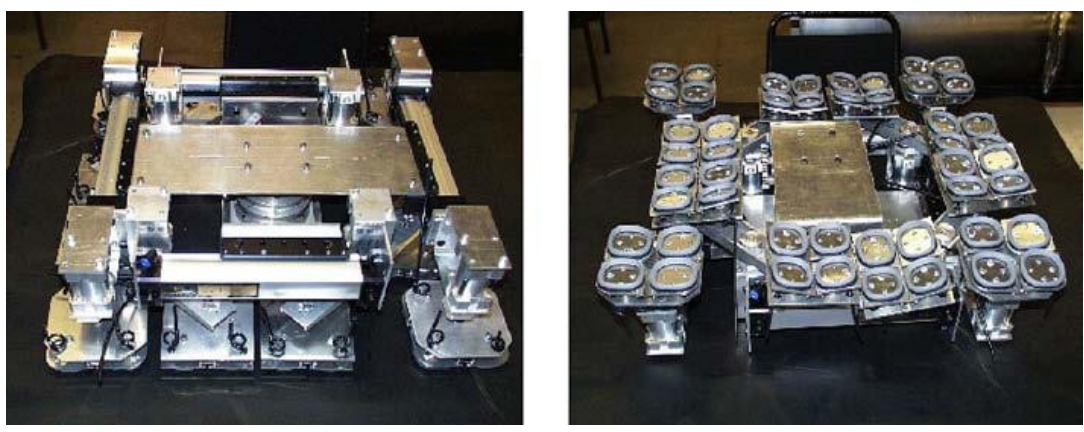

Figure 3 The RobAir climbing robot platform. ${ }^{1}$ 


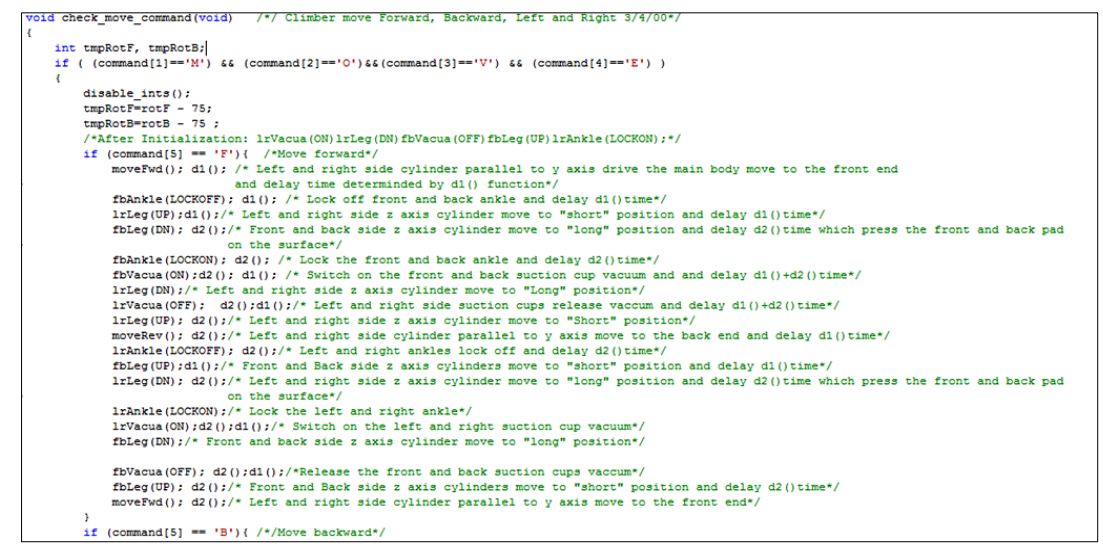

Figure 4 Extract codes from the control software of nuclear plant inspection robot shown in Fig2

working on a flat surface. It is clear that the smaller "a" would make the robot easy to work on different types of surfaces.

\section{The New Robot Design}

\subsection{Structure Design}

To overcome the disadvantages of pneumatic cylinder driven climbing robots, we have designed a new range of climbing robot which are actuated by electric motors. The new design uses rotation to drive robot to move forward or backward. The robot size can be very small and will be much faster and more

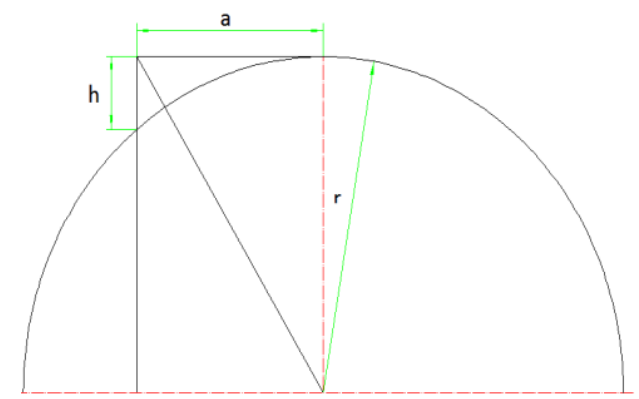

Figure 5 Different working surface requires different length of climbing robot legs. 


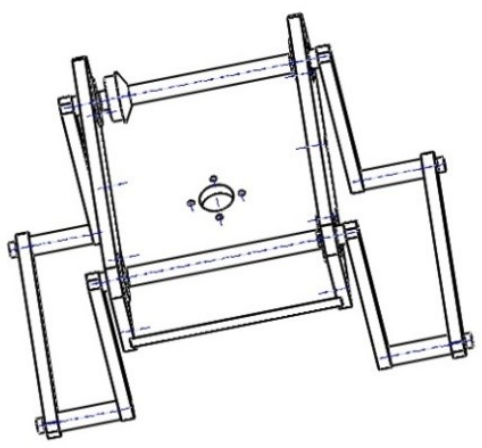

(a)

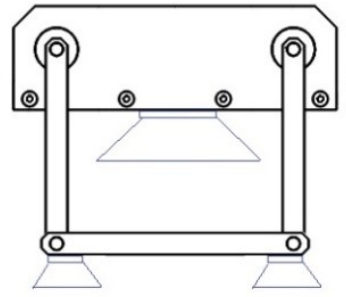

(b)

Figure 6 The 3D view and side view of the new robot mechanism.

agile compared with the cylinder driven climbing robots.

The overall new robot mechanism is shown in Figure 6. Fig 6(a) is the 3D image of the mechanism. Fig 6(b) is the side view and it shows the large suction cup (FC100P $100 \mathrm{~mm}$ diameter) installed.

Four small suction cups (ZPT-UC-50-6-A10, 50mm diameter) are installed to the side legs. The size of the robot platform is $150 \times 108 \mathrm{~mm}$ which is much smaller compare with RobAir $(518 \times 518 \mathrm{~mm})$. There are two degrees, one is the rotation of the legs and one is rotation of the central suction cups. The rotation of the legs will drive the side cups to make a circular movement parallel to the robot main body platform. It will drive the robot move forward or backward. The rotation degree of the central part will be sued to change the direction of the

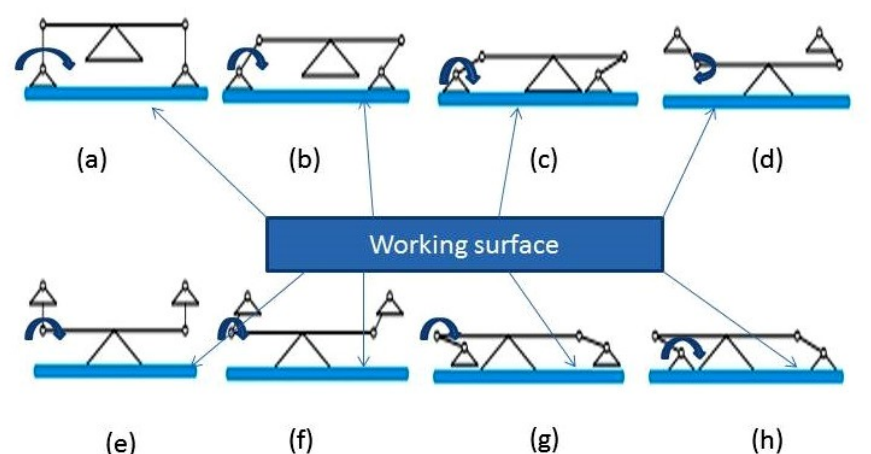

Figure 7 Examples of the new robot movement sequences 


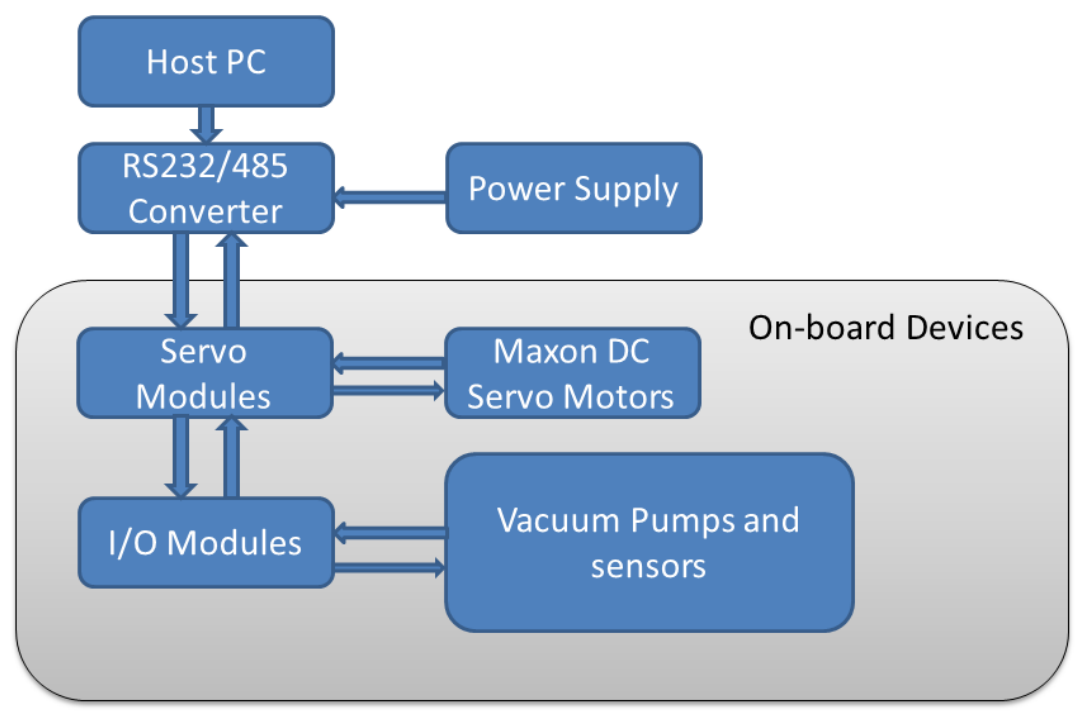

Figure 8 Control diagram.

robot movement.

Figure 7 shows the movement sequences of the newly designed robot vehicle. In Fig 7(a), the side cups vacuum switched on and suck to the surface, the motor driven side leg will move the robot platform forward to Fig. 7(b); continue rotating until the central cup contact the surface. Stop the rotation of side legs and switch on the vacuum of central cup, then switch off the side cups vacuum and rotating the side leg in the same direction.

The platform will stop to move and the leg rotation will cause the side suction cups to move circularly through the status of Fig. 7(d), (e), (f), (g) until (h) when the side cups will touch the surface, then stop the leg rotation and switch on the side cups vacuum, then release the central cup, further rotating the side leg will drive the robot platform to move forward like described earlier. If the robot wants to change the direction, this can be achieved when the central cup is stuck to the working surface. It could be when the robot is in the status like Fig 7(e) or during the leg movement in the air.

\subsection{Control system design}

The control system (Fig. 8) is based on author's work on the Robtank ${ }^{2}$ and RobAir ${ }^{1}$ projects. The on-board DC motor drivers and I/O modules developed in 
RobTank project ${ }^{3}$ are used to control the motors and the valves, and at same time to receive the feedback from sensors. The communication between the host computer and the on-board modules is via RS485 connections. It has been proved to be successful in the RobTank and RobAir projects and also for this system. The advantage of this design has been discussed in paper. ${ }^{1-3}$

\subsection{The new Design}

Figure 9 shows the photo taken from different sides of the first prototype of

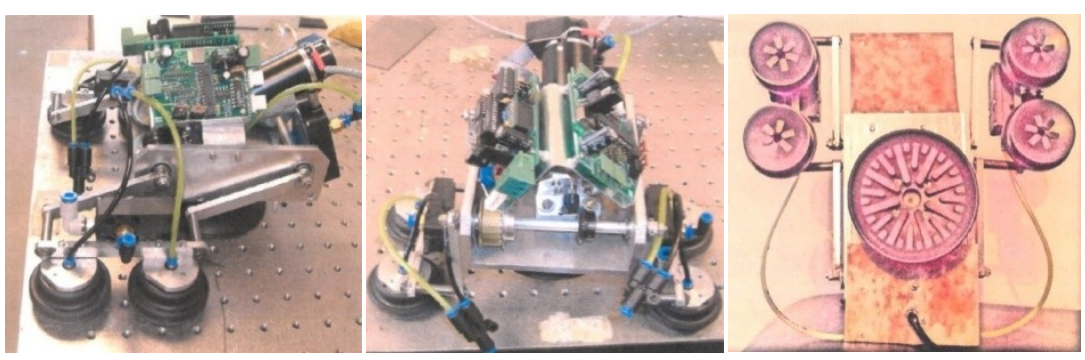

Figure 9 Photos of the new designed robot in different views.

this robot. The central suction cap has the diameter of $100 \mathrm{~mm}$ and the side cups each have a diameter of $50 \mathrm{~mm}$. This means that the suction force of side cups and the central cups are almost same. The lifting forces are displayed in table 1, from PIAB's website. ${ }^{4}$ The first prototype weighs less $3 \mathrm{~kg}$. It incorporates the robot payload range of $10-30 \mathrm{~kg}$ which is very good compared with the robots

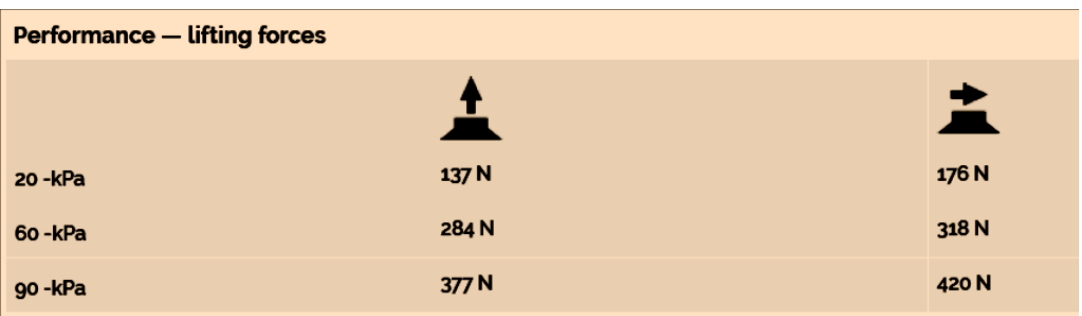

Table 1 PIAB suction cup FC100P performance table. ${ }^{4}$ 
mentioned earlier. The tests show that the robot can walk very well on a flat surface and surfaces with small curve.

\section{Conclusion}

The design of a new structure mechanism climbing robot that uses the DC motor combine with the rotation leg to replace the traditional pneumatic cylinder actuator can make the robot to have a miniature size, which further simplify the moment sequence and able to work in different type of working surface. It can used to replace human being carrying NDT sensors for inspection work in hazard environment. The author are working on develop the portable NDT device which can be carried by this robot. The further improvement of the leg part of this robot is under the way.

\section{References}

1. Janzhong Shang, Tariq P Sattar, Shuwo Chen and Bryan Bridge Design of a climbing robot for inspecting aircraft wings and fuselage, Industrial Robot, 34(6):495-502 - October 2007

2. Sattar T.P., Zhao Z., Feng J., Bridge B., Mondal S., Chen S., (2002) Internal In-service Inspection of the floor and walls of Oil, Petroleum and Chemical Storage Tanks with a Mobile Robot, Proc. Of 5th International Conference on Climbing and Walking Robots and the Support Technologies for Mobile Machines, ISBN 186058380 6, 2002, pp 947-954, Professional Engineering Publishing Ltd. UK

3. Shuwo Chen, Jianzhaong Shang, Zhanfang Zhao Novel Solutions to Design Problems of Industrial Climbing Robots, Proceedings of the 8th International Conference on Climbing and Walking Robots and the Support Technologies for Mobile Machines, CLAWAR 2005, London, UK, September 13-15, 2005

4. https://www.piab.com/Products/suction-cups/shape/flat-concave/fc---flatconcave-20-150-mm/0103297/ (accessed $1^{\text {st }}$ May 2017) 